International Archives of the Photogrammetry, Remote Sensing and Spatial Information Sciences,

Volume XL-7/W1, 3rd ISPRS IWIDF 2013, 20 - 22 August 2013, Antu, Jilin Province, PR China

\title{
A NOVEL IMAGE REGISTRATION ALGORITHM FOR SAR AND OPTICAL IMAGES BASED ON VIRTUAL POINTS
}

\author{
Cuifang $\mathrm{Ai}^{\mathrm{a}}$, Tiantian Feng ${ }^{\mathrm{a}}$, Jianmei Wang ${ }^{\mathrm{a}}$, Shaoming Zhang ${ }^{\mathrm{a}}$ \\ College of Surveying and Geo-Informatics, Tongji University, 1239 Siping Road, Shanghai, China- \\ aicuifang123@163.com
}

KEY WORDS: image registration, SAR image, optical image, region feature, virtual points

\begin{abstract}
:
Optical image is rich in spectral information, while SAR instrument can work in both day and night and obtain images through fog and clouds. Combination of these two types of complementary images shows the great advantages of better image interpretation. Image registration is an inevitable and critical problem for the applications of multi-source remote sensing images, such as image fusion, pattern recognition and change detection. However, the different characteristics between SAR and optical images, which are due to the difference in imaging mechanism and the speckle noises in SAR image, bring great challenges to the multi-source image registration. Therefore, a novel image registration algorithm based on the virtual points, derived from the corresponding region features, is proposed in this paper. Firstly, image classification methods are adopted to extract closed regions from SAR and optical images respectively. Secondly, corresponding region features are matched by constructing cost function with rotate invariant region descriptors such as area, perimeter, and the length of major and minor axes. Thirdly, virtual points derived from corresponding region features, such as the centroids, endpoints and cross points of major and minor axes, are used to calculate initial registration parameters. Finally, the parameters are corrected by an iterative calculation, which will be terminated when the overlap of corresponding region features reaches its maximum. In the experiment, WordView- 2 and Radasat- 2 with $0.5 \mathrm{~m}$ and $4.7 \mathrm{~m}$ spatial resolution respectively, obtained in August 2010 in Suzhou, are used to test the registration method. It is shown that the multi-source image registration algorithm presented above is effective, and the accuracy of registration is up to pixel level.
\end{abstract}




\section{INTRODUCTION}

With the enormous increase in availability and quality of remote sensing data products, remote sensing imagery and techniques have found applications in diverse areas like traffic studies, treaty and border monitoring, agricultural studies, generation of 3-D models and topographic maps, early-warning systems, urban growth monitoring, damage assessment, and specifically, disaster mitigation [5]. In general, images acquired both by the passive optical sensors and active synthetic aperture radar sensors (alone and in combination) are major sources for crisis information management. Optical image is rich in spectral information, while SAR instrument can work in both day and night and obtain images through fog and clouds. Combination of these two types of complementary images shows the great advantages of better image interpretation. Image registration refers to the task of aligning two or more images of the same scene taken under different imaging conditions, such as at different times, by different sensors, from different viewpoints, etc. It is a crucial step in combination of this two types of complementary images.

Automatic registration falls into two categories: intensity-based method and feature-based method. The intensity-based method finds the geometric transformation between optical and SAR images by optimizing a similarity measure between the two images[2]. Widely used measures include mutual information, cluster reward algorithm, and cross-cumulative residual entropy .The intensity-based methods work directly on image intensities, are very sensitive to image noise, and will become unreliable when they exist significant grey level difference and images distortion between the both images[7]. Thus, these methods are not suitable for multi-sensor images registration, not to mention for optical and SAR images. The feature-based method usually estimates the geometric transformation between images by establishing reliable feature matches. SAR image mainly reflects microwave backscatter coefficient of the targets, while optical image primary record solar radiation. Featurebased method tend to achieve better result compared with intensity-based method because of the difference in imaging mechanism and the speckle noises in SAR image.

For these features, point features and line features are usually huge in number and easy to locate, but difficult to match, especially when they are from different sensors. The number of closed regions is usually small, but they are relatively reliable and easy to match. For SAR and optical images, a novel image registration algorithm based on the virtual points, derived from the corresponding region features, is proposed in this paper.

\section{METHODS}

Feature-based methods basically consist of four steps: feature extraction, feature matching, transform model estimation and image transformation.

\subsection{Feature extraction}

Many closed regions extracting methods have successfully developed in the past decades, such as Chan-Vese model, GAC model, morphological segmentation algorithm, dynamic threshold technique, region increase method, the mean-shift algorithm etc . However, the speckle noise strongly destroying contours and boundaries of the objects in SAR images, the above methods are not only time consuming but cannot obtain a very good effect.

Among variety of objects, water is the most easily recognizable and could extract reliable regions in common. In this paper, the main closed regions are coming from the classification of water. Firstly, Lee filter is used to eliminate the speckle noise of SAR images. Then unsupervised classification IsoDate algorithm and supervised classification algorithm are adopted to extract closed regions from SAR and optical images simultaneously. After closed region extracting, there are still many small regions and noise. So morphological operations and threshold method are used to avoid this kind of noise and improve the connectedness of the objects.

\subsection{Feature matching}

For the extracted closed regions, reasonable description and comparison of its characteristics are used to identify matching relations. Reasonable shape description parameters contain area, perimeter, the length of major and minor axes. The feature matching achieved by the cost function is defined as (1).

$$
C_{\mathrm{ij}}=\left|\frac{s_{i}-s_{j}}{s_{i}+s_{j}}\right|^{\frac{1}{2}}+\left|\frac{p_{i}-p_{j}}{p_{i}+p_{j}}\right|+\left|\frac{r_{i}-r_{j}}{r_{i}+r_{j}}\right|+\left|\frac{c_{i}-c_{j}}{c_{i}+c_{j}}\right|
$$

Where $S_{i}$ is the area of region $i, p_{i}$ is the perimeter length, $r_{i}$ is the length of the bounding rectangle, and $c_{i}$ is the width of the bounding rectangle.

Only when two conditions to be satisfied, feature $\mathrm{S}$ comes from SAR image and feature $\mathrm{O}$ comes from optical image are determined to be matched:

$(1) \mathrm{C}_{\mathrm{so}}=\min \left(\mathrm{C}_{\mathrm{so} 1}, \mathrm{C}_{\mathrm{so} 2}, \ldots, \mathrm{C}_{\mathrm{son}}\right)$ where $\mathrm{O}_{1}, \mathrm{O}_{2}, \ldots, \mathrm{O}_{\mathrm{n}}$ are the possible matched features to feature $\mathrm{S}, \mathrm{n}$ is the number of closed regions in the optical image.

\section{(2) $\mathrm{C}_{\text {so }} \leqq T$, which $T$ is threshold value. If the cost function is}

less than the threshold value, the corresponding features will be eliminated.

\subsection{Transform model estimation}

Affine transformation is widely used in image registration and supports modeling of translation, rotation, stretching, and shearing [6]. The goal is to seek an appropriate transformation which correctly maps a position $\left(\mathrm{x}^{\prime}, \mathrm{y}^{\prime}\right)$ on the optical image to a position $(\mathrm{x}, \mathrm{y})$ in the SAR image. As satellite images are very far from the camera sensor, the projective transformation can be approximated by the affine transformation involving six parameters.

$$
\begin{aligned}
x^{\prime} & =m_{1} x+m_{2} y+m_{3} \\
y^{\prime} & =m_{4} x+m_{5} y+m_{6}
\end{aligned}
$$

Virtual points derived from corresponding region features $\mathrm{Cs}_{\mathrm{i}} \mathrm{O}_{\mathrm{j}}$ got in the above step, such as the centroids, endpoints and cross points of major and minor axes, are used to calculate initial registration parameters. Then, the parameters are corrected by an iterative calculation, which will be terminated when the overlapping of corresponding region features reaches its maximum.

\subsection{Image transformation and resampling}

The result of last three processing steps is the optimal six transformation parameters. Affine transformation in Eq. (2)(3) are used based on those parameters. After transformation, chose bi-cubic convolution interpolation method to resample the sense image. 

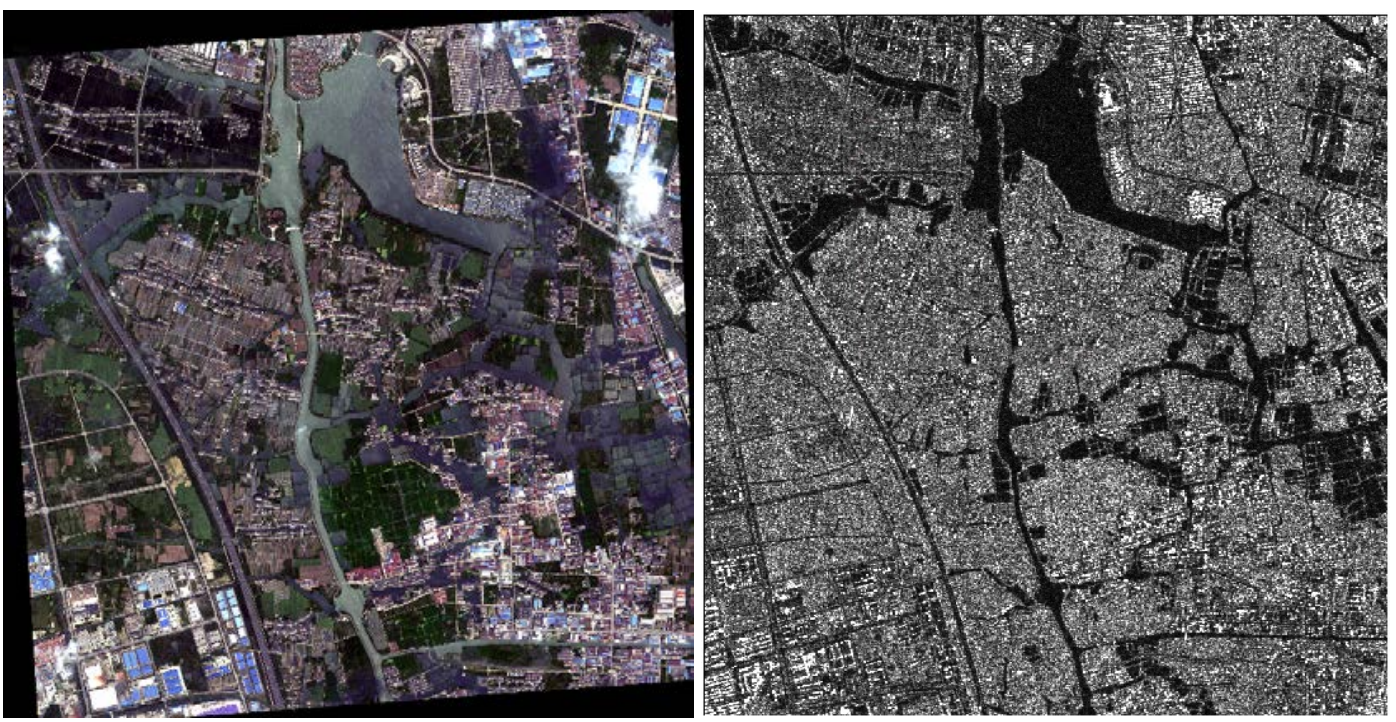

Figure 1. The original optical and SAR images
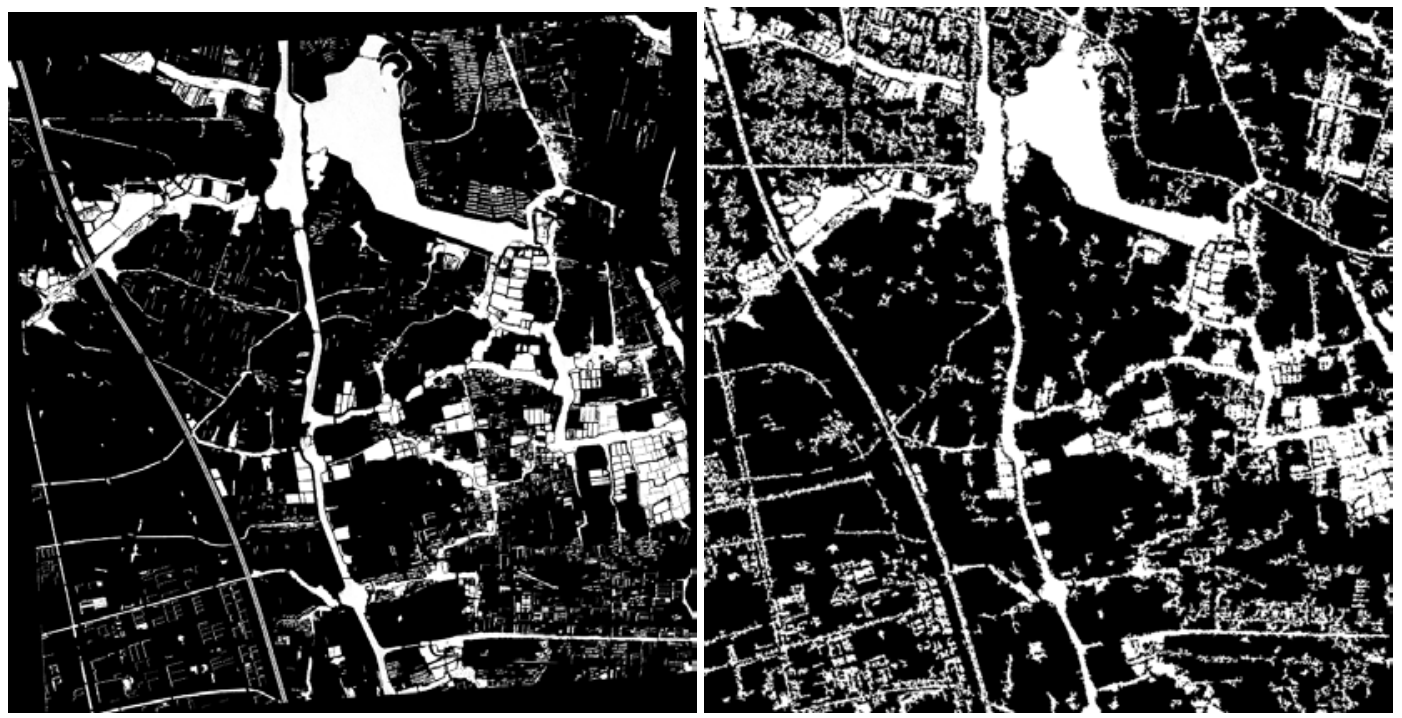

Figure 2. Closed regions extraction results

\section{EXPERIMENTS}

In our experiments, the optical image $10591 \times 10804(0.5 \mathrm{~m})$ was taken from WordView- 2 and the SAR image $1116 \times 1138(4.7 \mathrm{~m})$ was taken from Radasat-2. They were all obtained in August 2010 in Suzhou. The original optical and SAR images are shown in Fig.1.

The Fig. 2 shows the closed regions extraction of optical and SAR images.

To estimate the accuracy of the registration results using the proposed method, all the valid CPs are taken into evaluation. Root Mean Square Error (RMSE) involving all the CPs is 0.972 pixels in the $\mathrm{X}$ direction and 0.854 pixels in the $\mathrm{Y}$ direction.

\section{CONCLUSIONS}

In this paper, we have presented a novel virtual points based approach to register optical image and SAR image. The virtual points derived from corresponding region features is used in affine transform model to obtain the initial parameters. Fine registration is carried out by maximizing the overlapping of the closed regions coming from the two images. And the experimental results shows that the virtual points based image registration algorithm presented above is effective, and the accuracy of registration is up to pixel level.

\section{REFERENCES}

[1]Zhenhua Wang,.Automatic registration of SAR and optical image based on multi-features and multiconstraints.ISPRS.2010.

[2]Bin Fan, Registration of Optical and SAR Satellite Imagesby Exploring the Spatial Relationshipof the Improved SIFT. IEEE GEOSCIENCE AND REMOTE SENSING LETTERS, VOL. 10, NO. 4, JULY 2013.

[3]Muhammad Adnan Siddique, Automatic registration of SAR and optical images based on mutual information assisted Monte Carlo.IGARSS 2012

[4]Lei Huang, SAR and optical images registration using shape context. IGARSS 2010

[5]Sahil Suri and Peter Reinartz, Mutual-Information-Based Registration of TerraSAR-X and Ikonos Imagery in Urban Areas. IEEE TRANSACTIONS ON GEOSCIENCE AND REMOTE SENSING, VOL. 48, NO. 2. 
International Archives of the Photogrammetry, Remote Sensing and Spatial Information Sciences, Volume XL-7/W1, 3rd ISPRS IWIDF 2013, 20 - 22 August 2013, Antu, Jilin Province, PR China

[6]Mahmudul Hasan, Multi-modal Registration of SAR and Optical Satellite Images.Digital Image Computing: Techniques and Applications.2009

[7] Jia Weijie, Automatic registration of SAR and optics image based on multi-features on suburban areas. Urban Remote Sensing Joint Event. 2009.

[8]Wei Shi, A visual circle based image registration algorithm for optical and SAR imagery.IGARSS.2012.

[9]Peng Wang, A Coarse-to-Fine Matching Algorithm for FLIR and optical satellite image registration. IEEE GEOSCIENCE AND REMOTE SENSING LETTERS, VOL. 9, NO. 4, JULY 2012.

[10]Hongsheng Li, Object matching using a locally affine invariant and linear programming techniques.IEEE Transaction on pattern analysis and machine intelligence,VOL.35,NO.2, FEBRUARY 2013. 